\title{
GA Design of Wire Pre-Fractal Antennas and Comparison With Other Euclidean Geometries
}

\author{
M. Fernández Pantoja, F. García Ruiz, A. Rubio Bretones, Senior Member, IEEE, \\ R. Gómez Martín, Senior Member, IEEE, J. M. González-Arbesú, \\ J. Romeu, Member, IEEE, and J. M. Rius, Member, IEEE
}

\begin{abstract}
A multi-objective genetic algorithm (GA) has been applied in conjunction with the numerical electromagnetic code (NEC) to the optimization of wire pre-fractal Koch-like antennas in terms of bandwidth, efficiency, and electrical size. Their performance is compared to that of other nonfractal designs.
\end{abstract}

Index Terms-Fractal wire antennas, genetic algorithms (GA).

\section{INTRODUCTION}

$\mathbf{T}$ HE design of electrically small antennas is a topic of current interest connected with the growing development of mobile-communication devices that require their components to be ever smaller and lighter.

As reducing the electrical size of an antenna is at the expense of other parameters such as bandwidth and efficiency [1], antenna miniaturization techniques require a compromise between all the parameters involved. This fact makes optimization techniques such as genetic algorithms (GA) a very appropriate tool for the design of such antennas. With this aim several authors have applied GA methods; for example Altshuler [2], who found a wire-resonant antenna presenting a maximum bandwidth for a given antenna size, and Choo et al. [3], who applied a multi-objective GA to design small wire antennas, taking into account bandwidth and efficiency.

Fractal shaped antennas, or more precisely, pre-fractal geometries that are built with a finite number of iterations due to their intricate and convoluted configurations, have been investigated by a number of authors as electrically small antennas [4]. GA techniques have also been applied to the design of prefractal antenna elements. For example a GA-engineered secondorder Koch-like dual antenna, having a compact size and low voltage standing wave ratio (VSWR) is presented in [5] and [6].

In this letter, we extend the work in [5] to the design, using a multi-objective GA of wire pre-fractal Koch-like antennas, seeking an optimum set of solutions in terms of resonance frequency, bandwidth, and efficiency. Moreover, the GA code is employed to search for nonfractal structures, namely zig-zag

\footnotetext{
Manuscript received June 16, 2003; revised July 25, 2003. This work was supported in part by the Spanish Projects TIC-2001-3236-C02-01, TIC-20012364-C03-01, TIC-2001-2364-C03-03, and by the European Project IST-200133055.

M. Fernández Pantoja, F. García Ruiz, A. Rubio Bretones, and R. Gómez Martín are with the Facultad de Ciencias, Department of Electromagnetics, University of Granada, 18071 Granada, Spain (rgomez@ugr.es).

J. M. González-Arbesú, J. Romeu, and J. M. Rius are with the Department Teoria del Senyal i Comunicacions, University of Politecnica Catalunya (UPC), 08034 Barcelona, Spain.

Digital Object Identifier 10.1109/LAWP.2003.819694
}

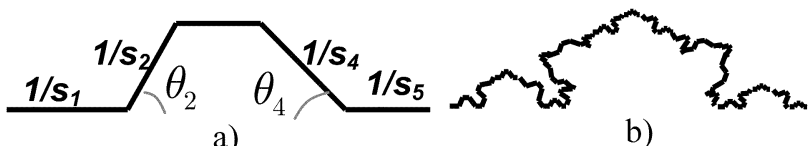

a)

b)

Fig. 1. Geometry of the (a) generating antenna and (b) its third-order iteration.

and meander type antennas, and the resulting optimized solutions are compared to those obtained when the search space is restricted to pre-fractal Koch-like elements. It is shown that for a given overall wire length and antenna size, GA find Euclidean geometry designs that perform better than their pre-fractal counterparts. These results are in consonance with the analysis presented in [7]-[9]. In our case, the use of a multi-objective GA permits a thorough search of possible designs fulfilling all the specifications and their comparison in terms of more parameters than just the resonance frequency.

\section{GA DESIGN}

\section{A. Design of Pre-Fractal Antennas}

An iterated function system (IFS) algorithm [5] is applied to generate the shape of the pre-fractal antenna elements to be considered as individuals in the initial GA population. An IFS is a superposition of affine transformations characterized by a finite number of parameters. Examples of generating a Koch curve by means of an IFS can be found in [5] and [10]. Fig. 1(a) shows an example of the kind of generating antennas considered in this paper, characterized by the parameters $\mathrm{s} 1, \mathrm{~s} 2, \mathrm{~s} 4, \mathrm{~s} 5, \theta_{2}$, and $\theta_{4}$, while Fig. 1(b) plots the resulting shape after the third order iteration of the IFS procedure.

A Pareto GA tool [11] has been developed to optimize several characteristics of the pre-fractal wire antennas simultaneously. In the GA code, the parameters s1, s2, s4, s5, $\theta_{2}, \theta_{4}$, which together with the number of iterations define a specific individual, are encoded into a chromosome. Instead of binary coding, fixed-point decimal coding has been chosen [11] to gain a better definition of the search space with a minimum computational cost. A set of monopole antennas that constitute the initial generation are randomly formed. They are fed at their base and their input impedance and resonance frequency are calculated applying the frequency-domain method of moments-based NEC code. To measure the goodness of a given individual and to permit the algorithm to evolve toward an optimal design, the following three fitness functions are defined:

$$
F_{i}^{1}=1-L_{i} ; F_{i}^{2}=\frac{\mathrm{BW}_{i}}{\Delta f} ; F_{i}^{3}=1-\frac{f_{r, i}}{f_{r}^{s}}
$$



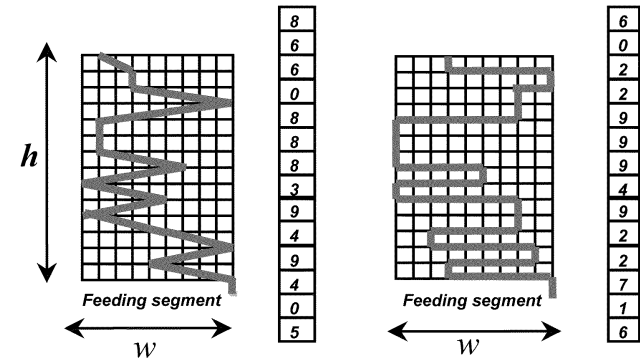

Fig. 2. Example of a zig-zag and a meander-type antenna.

where $L_{i}$ is the length of the stretched wire corresponding to antenna $i, \mathrm{BW}_{i}$ the $2: 1 \mathrm{SWR}$ bandwidth, $\Delta f$ the frequency range of interest, $f_{r, i}$ the resonance frequency of antenna $i$, and $f_{r}^{s}$ the resonance frequency of a straight monopole of length $h$. Our aim is to maximize $F^{1}$ and therefore minimize the ohmic losses that are related to length, $F^{2}$ so that a maximum bandwidth is achieved, and $F^{3}$ so that for a given size of the antenna its resonance frequency is the smallest possible and, therefore, so is its electrical size at resonance. The joint maximization of the three parameters in (1) contributes to obtaining individuals with a low $\mathrm{Q}$ factor and a maximum efficiency. In order to avoid convergence to impractical individuals, constraints are also incorporated into the fitness functions and a penalty [12] is imposed on those individuals that while not unfeasible as generating antennas, would be impossible to realize at a higher order. Any antenna is also restricted to fit in a given rectangle of dimensions $h \times w, w$ being the width of a Koch curve of height $h$.

After applying the GA operators [11] of tournament method, one point crossover and a Gaussian probability-distribution mutation, the multi-objective GA procedure renders a front or surface of optimal solutions (Pareto front) from which the designer can select the individual that best fits the requirements of the problem at hand. The procedure is applied by means of domination schemes using triangular sharing functions to guarantee diversity in the final set of optimal solutions [11].

\section{B. Euclidean Antennas}

In order to investigate whether fractal shapes might be the best alternative for the design of efficient antennas with minimum resonance frequency, we have also allowed our GA code to look for Euclidean geometries. In particular, zig-zag and meander-type monopole antennas have been considered. An example of a zig-zag and a meander-type antenna formed, respectively, by 14 and 24 segments, is shown in Fig. 2, together with their corresponding 14-bit decimal-coded chromosomes.

\section{RESULTS}

The specific GA adopted in this work employs both a crossover operator and a mutation operator with probabilities $80 \%$ and $5 \%$, respectively. The population is formed with 20 chromosomes, comprising a set of $\mathrm{N}=12$ genes which represent coded versions of the individual characteristics.

We begin by designing several wire antennas optimizing with GA only one at a time of the cost functions given in (1). In all cases, the possible antenna designs are restricted to the rectangle of height $h=6.22 \mathrm{~cm}$ and width $w=1.73 \mathrm{~cm}$. Eu-

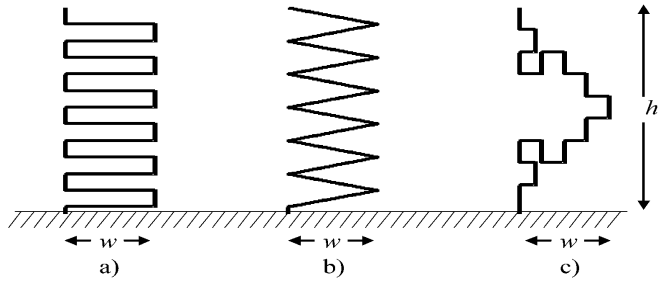

Fig. 3. (a) Meander-type antenna $\mathrm{L}=26.97 \mathrm{~cm}, f_{r}=449.8 \mathrm{MHz}, \mathrm{BW}=$ $164 \mathrm{MHz}$. (b) Zig-zag type antenna $\mathrm{L}=22.01 \mathrm{~cm}, f_{r}=559 \mathrm{MHz}, \mathrm{BW}=$ $222 \mathrm{MHz}$. c) Koch-like antenna $\mathrm{L}=15.52 \mathrm{~cm}, f_{r}=665.5 \mathrm{MHz}, \mathrm{BW}=$ $276.3 \mathrm{MHz}$.

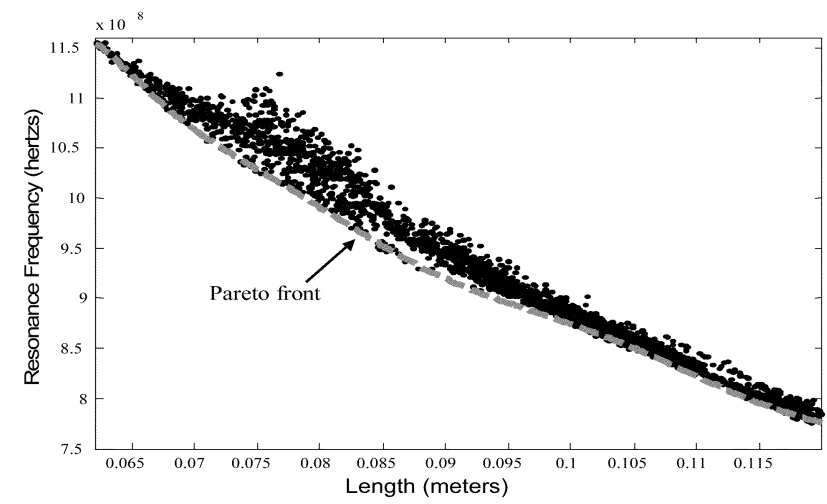

Fig. 4. Resonance frequency versus length of each Koch-like antenna at each iteration of the GA process. Dashed line: Pareto front.

clidean geometries are formed by 12 (zig-zag) or 24 (meander) wire segments (in both cases $N=12$ ) and Koch-like antennas are considered up to second order. The best solution in terms of minimum length was the straight wire monopole of length $6.22 \mathrm{~cm}$. The same monopole was again found by GA when maximum bandwidth was requested. These are logical results that help to validate our GA code and that were obtained irrespective of whether the GA code was allowed to search for Euclidean or Koch-like solutions. Regarding minimum resonance frequency (maximum $F^{3}$ ), Fig. 3 shows the optimum second-order Koch-like solution [Fig. 3(a)], the zig-zag type solution [Fig. 3(b)], and the meander-type solution [Fig. 3(c)]. The total length, L, the resonance frequency, $f_{r}$, and the bandwidth (BW) of these antennas are given in the figure caption. Note that the antenna having minimum $f_{r}$ (the meander) presents poor bandwidth and high length, while the pre-fractal design having lowest ohmic losses and widest $\mathrm{BW}$, has a worse $f_{r}$ than both Euclidean designs. This is clearly a problem for which a multi-objective GA is needed in order to seek a compromise between the different parameters and to compare different designs under the same conditions.

Next, multi-objective optimization is considered in which we seek antennas, either second-order Koch-like or Euclidean, that fitting in the rectangle $6.22 \mathrm{~cm} \times 1.73 \mathrm{~cm}$ presents minimum resonance frequency, and length. Fig. 4 shows, with dot symbols, a plot of resonance frequency versus length for each individual and each iteration of the GA process for the case of a Koch-like design after 350 generations. As GA progresses, the envelope of the graph evolves to an optimal set of solutions, called the Pareto front, which is shown as a dashed line in Fig. 4 and corresponds to the result after 2000 generations. Fig. 5 


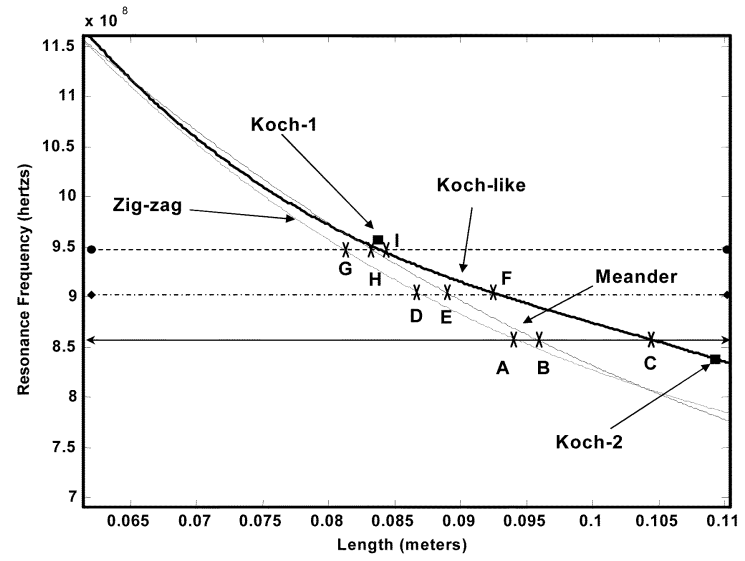

Fig. 5. Pareto fronts for the meander, zig-zag, and Koch-like cases when optimizing $F^{1}$ and $F^{3}$.

TABLE I

RESONANCE FREQUENCY, LENGTH, BANDWIDTH AND INPUT RESISTANCE OF THE INDIVIDUALS MARKED WITH LETTERS IN FIG. 5

\begin{tabular}{c|c|c|c|c}
\hline & $\mathrm{f}_{\mathrm{r}}(\mathrm{MHz})$ & $\mathrm{L}(\mathrm{cm})$ & $\mathrm{BW}(\mathrm{MHz})$ & $\mathrm{R}_{\text {in }}(\mathrm{ohms})$ \\
\hline $\mathrm{A}$ & 852.81 & 9.47 & 360.87 & 23.44 \\
\hline $\mathrm{B}$ & 854.89 & 9.64 & 367.11 & 23.23 \\
\hline $\mathrm{C}$ & 865.49 & 10.22 & 360.53 & 20.11 \\
\hline $\mathrm{D}$ & 900.21 & 8.74 & 382.38 & 28.44 \\
\hline $\mathrm{E}$ & 900.07 & 8.98 & 385.45 & 25.79 \\
\hline $\mathrm{F}$ & 904.95 & 9.33 & 379.86 & 22.63 \\
\hline $\mathrm{G}$ & 953.98 & 7.92 & 407.63 & 30.60 \\
\hline $\mathrm{H}$ & 960.75 & 7.95 & 410.14 & 26.36 \\
\hline $\mathrm{I}$ & 957.3 & 8.25 & 405.36 & 26.10 \\
\hline
\end{tabular}

also includes the Pareto front of optimal solutions in terms of length and resonance frequency for the cases of zig-zag and meander-type antennas. The first and second iterations of a Koch monopole are also plotted for reference. It can be seen that Euclidean geometries seem to perform better than the pre-fractal designs in reducing $f_{r}$ given that for most of the possible lengths they render resonance frequencies that are smaller than those of their pre-fractal counterparts. Just at very low values of $\mathrm{L}$ all the antennas present a similar behavior, but it should be mentioned that the optimization process did not provide enough number of individuals in this specific range of length for the results to be significant. For lengths higher than $10.5 \mathrm{~cm}$, meander antennas seem to be the electrically smallest choice. It should be pointed out that although, to minimize computational resources, we did not search for greater lengths or pre-fractal orders, the results in Fig. 5 show that the distance between the Pareto fronts corresponding to Euclidean and pre-fractal designs increases with length, presenting the Euclidean antennas lower values of $f_{r}$.

In order to compare the performance of different geometries that have approximately the same $f_{r}$, several individuals are selected from Fig. 5 (see the horizontal lines that mark individuals A-I). Their corresponding parameters are given in Table I confirming the better behavior of Euclidean antennas.

Next, the bandwidth and length are considered. Fig. 6 shows the Pareto fronts corresponding to second-order pre-fractal, zig-zag, and meander-type antennas when the optimized parameters are $F^{1}$ and $F^{2}$. Again the Koch 1 and 2 results have

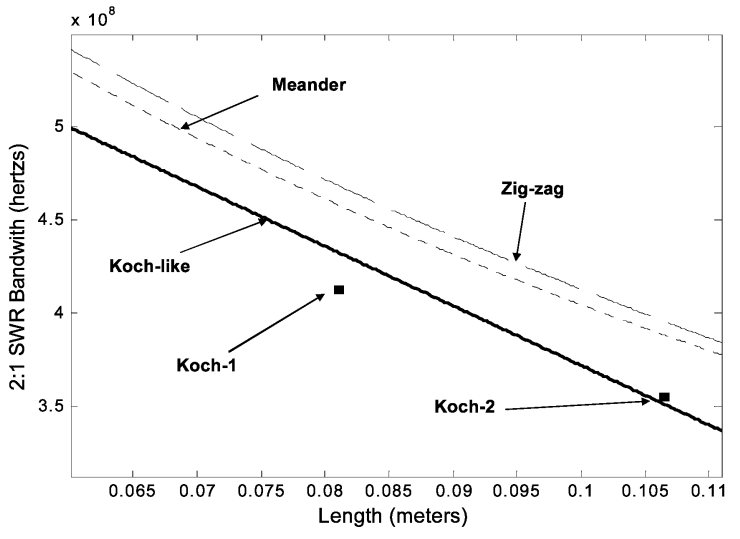

Fig. 6. Pareto fronts for the meander-, zig-zag, and Koch-like cases when the parameters optimized are $F^{1}$ and $F^{2}$.

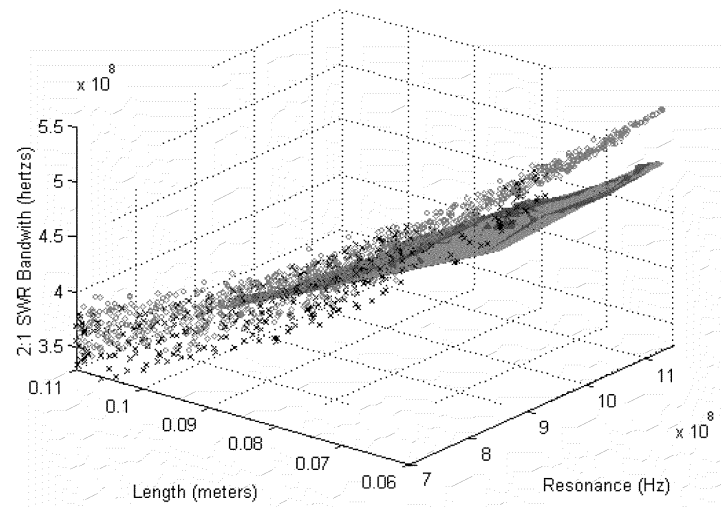

Fig. 7. Pareto surfaces when optimizing $F^{1}, F^{2}$ and $F^{3}$.

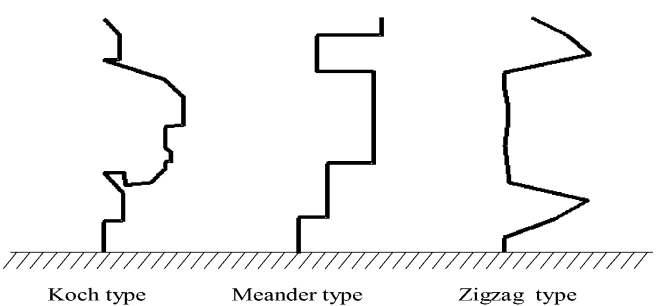

Fig. 8. Geometry of the three antennas selected from Fig. 7.

been marked for comparison. Note that both the meander- and the zig-zag-type show a better performance in terms of BW than the pre-fractal structures since, for a given length, they present the widest BW; the zig-zag-type antennas always being the best choice.

Finally, the three fitness functions (1) have been optimized at the same time, seeking an antenna design fitting in the same rectangle as in the previous examples. Now the Pareto fronts are the surfaces shown in Fig. 7. The squared and crossed symbols represent the zig-zag and meander-type solutions, respectively, while Koch-like solutions are shown as a continuous surface. From that figure, the designer can choose the antenna elements that best fit the specified requirements of the problem at hand. In particular we have selected the individuals plotted in Fig. 8, all of them having approximately the same length $(L$; $10.22 \mathrm{~cm}$ ) and radius of $0.1 \mathrm{~mm}$. Their resonance frequencies $\mathrm{f}_{r}$, Q factor, bandwidth and efficiency $\eta$, calculated with NEC, 
TABLE II

PARAMETERS OF THE ANTENNAS SHOWN IN FIG. 8 CALCUlated Numerically

\begin{tabular}{l|l|l|l|l}
\hline Antenna & $\mathrm{f}_{\mathrm{r}}(\mathrm{MHz})$ & $\mathrm{Q}$ & $\eta(\%)$ & $\begin{array}{l}\mathrm{BW} \\
(\mathrm{MHz})\end{array}$ \\
\hline Koch & 864.5 & 13.57 & 96.8 & 365.4 \\
\hline Meander & 826.5 & 12.67 & 97.19 & 346.3 \\
\hline Zig-zag & 824 & 13.99 & 96.79 & 330.6 \\
\hline
\end{tabular}

TABLE III

EXPERIMENTAL RESULTS FOR THE ANTENNAS IN FIG. 8

\begin{tabular}{l|l|l|l}
\hline & $\mathrm{f}_{\mathrm{r}}(\mathrm{MHz})$ & $\mathrm{Q}$ & $\eta(\%)$ \\
\hline Koch & 905 & 12.67 & 87.64 \\
\hline Meander & 850 & 12.60 & 88.78 \\
\hline Zig-zag & 870 & 13.89 & 87.34 \\
\hline
\end{tabular}

are given in Table II. Experimental results are obtained when the antennas are modeled as copper strips $(0.29 \mathrm{~mm}$ width and $25 \mu \mathrm{m}$ thick) printed on a 0.25 -mm-thick fiberglass FR4. The dimensions of the strip are chosen so the actual geometry where the current flows in the strip has the same cross-section surface as when it flows in the original wire. The measured data, obtained following [13] are given in Table III and show a reasonable agreement with the numerical experiments confirming that the nonfractal antennas perform better than the fractal designs. The slight differences between measurements and numerical data can be attributed to the two different models considered for the antennas.

\section{CONCLUSION}

A multi-objective GA algorithm has been applied to the design of wire antennas optimizing their bandwidth and efficiency while reducing their resonance frequency. Second-order
Koch-like pre-fractal antennas have been considered as well as other Euclidean geometries such as zig-zag and meander-type antennas. The performance of the nonfractal curves was always better than that of the Koch-like structures.

\section{REFERENCES}

[1] L. J. Chu, "Physical limitations on omni-directional antennas," J. Appl. Phys., vol. 19, pp. 1163-1175, 1948.

[2] E. E. Altshuler, "Electrically small self-resonant wire antennas optimized using a genetic algorithm," IEEE Trans. Antennas Propagat., vol. 50, pp. 297-300, Mar. 2002.

[3] H. Choo, R. Rogers, and H. Ling, "Design of electrically small wire antennas using genetic algorithm taking into consideration bandwidth and efficiency," in Proc. IEEE AP-URSI Symp., San Antonio, TX, 2002.

[4] J. P. Gianvittorio and Y. Rahmat-Samii, "Fractal antennas: A novel antenna miniaturization technique, and applications," IEEE Antennas Propagat. Mag., vol. 44, pp. 20-36, Feb. 2002.

[5] D. H. Werner, P. L. Werner, and K. H. Church, "Genetically engineered multiband fractal antennas," Electron. Lett., vol. 37, no. 19, pp. 1150-1151, 2001

[6] D. H. Werner and S. Gaungly, "An overview of fractal antenna engineering research," IEEE Antennas Propagat. Mag., vol. 45, pp. 38-57, Feb. 2003.

[7] S. R. Best and J. D. Morrow, "The effectiveness of space-filling fractal geometry in lowering resonant frequency," IEEE Antennas Wireless Propagat. Lett., vol. 1, pp. 112-115, 2002.

[8] S. R. Best, "On the resonant properties of the Koch fractal and other wire monopole antennas," IEEE Antennas Wireless Propagat. Lett., vol. 1, pp. 74-76, 2002.

[9] P. L. Werner and D. H. Werner, "A design optimization methodology for multiband stochastic antennas," in Proc. IEEE AP-URSI Symp., vol. 2, San Antonio, TX, 2002, pp. 354-357.

[10] C. P. Baliarda, J. Romeu, and A. Cardama, "The Koch monopole: A small fractal antenna," IEEE Trans. Antennas Propagat., vol. 48, pp. 1773-1781, Nov. 2000.

[11] E. T. Back, Handbook of Evolutionary Computation. New York: Oxford Univ. Press, 1997.

[12] C. A. Coello, "Constraint-handling using an evolutionary multiobjective optimization technique," in Proc. Genetic and Evolutionary Computation Conf. (GECCO'99), Orlando, FL, 1999, pp. 117-118.

[13] W. E. McKinzie, "A modified wheeler cap method for measuring antenna efficiency," in Proc. IEEE Antennas and Propagat Symp., vol. 1, Montreal, QC, Canada, 1997, pp. 13-18. 